\title{
Advanced fluid-typing methods for NMR logging
}

\author{
Xie Ranhong ${ }^{1,2 *}$ and Xiao Lizhi ${ }^{1,2}$ \\ ${ }^{1}$ State Key Laboratory of Petroleum Resource and Prospecting, China University of Petroleum, Beijing 102249, China \\ ${ }^{2}$ Key Laboratory of Earth Prospecting and Information Technology, China University of Petroleum, Beijing 102249, China \\ (C) China University of Petroleum (Beijing) and Springer-Verlag Berlin Heidelberg 2011
}

Abstract: In recent years, nuclear magnetic resonance (NMR) has been increasingly used for fluidtyping in well-logging because of the improved generations of NMR logging tools. This paper first discusses the applicable conditions of two one-dimensional NMR methods: the dual $T W$ method and dual $T E$ method. Then, the two-dimensional $\left(T_{2}, D\right)$ and $\left(T_{2}, T_{1}\right)$ NMR methods are introduced. These different typing methods for hydrocarbon are compared and analyzed by numerical simulation. The results show that the dual $T W$ method is not suitable for identifying a macroporous water layer. The dual $T E$ method is not suitable for typing gas and irreducible water. $\left(T_{2}, T_{1}\right)$ method is more effective in typing a gas layer. In an oil-bearing layer of movable water containing big pores, $\left(T_{2}, T_{1}\right)$ method can solve the misinterpretation problem in the dual $T W$ method between a water layer with big pores and an oil layer. The $\left(T_{2}, T_{1}\right)$ method can distinguish irreducible water from oil of a medium viscosity, and the viscosity range of oil becomes wide in contrast with that of the dual $T W$ method. The $\left(T_{2}, D\right)$ method is more effective in typing oil and water layers. In a gas layer, when the SNR is higher than a threshold, the $\left(T_{2}, D\right)$ method can resolve the overlapping $T_{2}$ signals of irreducible water and gas that occurs due to the use of the dual TE method. Twodimensional NMR for fluid-typing is an important development of well logging technology.

Key words: NMR logging, one-dimensional NMR, two-dimensional NMR, reservoir evaluation, fluidtyping

\section{Introduction}

Nuclear magnetic resonance (NMR) technology, which has been widely used in well-logging and in petrophysics for core analysis, is crucial for oil and gas exploration and development. NMR logging can provide much information on porosity, capillary bound water, clay bound water, the volume of movable fluid, permeability, and pore size distribution (Coates et al, 1999; Dunn et al, 2002; Xie et al, 2006; Xie et al, 2008; Lu et al, 2007; He et al, 2005), all of which are important in well-logging for evaluating reservoirs, estimating water saturation, and understanding the fluid flow in pore networks, especially for prospecting and developing areas with a complex lithology. Akkurt and Prammer showed that NMR logging not only could provide porosity, bulk volume irreducible water, free fluids, permeability but also allow the identification and quantification of fluids in a reservoir. They suggested a dual $T W$ method based on the $T_{1}$ contrast between water and hydrocarbon and a dual $T E$ method based on the diffusion coefficient contrast between fluids (Akkurt et al, 1995; Prammer et al, 1995). These two NMR methods, which have been widely used for typing fluids in well-logging

*Corresponding author. email: xieranhong@cup.edu.cn

Received January 14, 2010 service companies of China, have generated some successful as well as unsuccessful examples (Tan et al, 2006; Yun and Tan, 2006). The latter sometimes even have results opposite to the prediction of oil and water zone interpretation (Xiao, 2007). In 2002, Sun and Dunn of Chevron Texaco Energy Technology Company, Hurlimann, Venkataramanan and Song et al of Schlumberger-Doll Research proposed independently a new concept of two-dimensional (2D) NMR (Sun and Dunn, 2002; Hurlimann et al, 2002; Song et al, 2002; Sun et al, 2004). This technical development gives rise to the expectation of better typing and evaluating reservoir fluids based on two-dimensional NMR logging. The basic problem with the theory and application of 2D NMR logging has been explored recently (Xie et al, 2005; Xie et al, 2009a; Xie and Xiao, 2009). This paper presents the applicable condition of typing fluids (oil, gas and water) with one-dimensional (1D) NMR methods, introduces the $\left(T_{2}, D\right)$ and $\left(T_{2}, T_{1}\right) 2 \mathrm{D}$ NMR methods, and discusses the advantage and limitation of different methods by numerical simulation.

\section{Theoretical basis of NMR logging typing fluids}

The differences in $T_{1}, T_{2}$ and $D$ among the main fluids in well-logging (oil, gas and water) in a reservoir condition form a theoretical basis for NMR fluid-typing, since the NMR 
properties of different reservoir fluids are quite different from one another. Table 1 is an example of $T_{1}, T_{2}$ and $D$ of reservoir fluids in a field gradient of $17 \mathrm{Gauss} / \mathrm{cm}$ with a TE of $1.2 \mathrm{~ms}$ for a typical sandstone reservoir (Dunn et al, 2002). The properties of these fluids are quite different. For example, $T_{1}$ for oil and gas is much longer than $T_{1}$ for water in the rock, which is the scientific basis for the dual $T W$ method. Diffusion coefficients are also quite different among oil, gas and water, which form the basis of the dual TE measurements. When multiple pore fluids, such as oil, gas and water, are present, it can become difficult to differentiate them, especially when their $T_{2}$ signals overlap due to a change of NMR properties of reservoir fluids, various crude oils components, wide viscosity ranges (Xie et al, 2007) and complex rock pore structures (He et al, 2005). Consequently, the prediction of oil and water zone interpretation can be inconsistent with the results from 1D NMR fluid- typing (Xiao, 2007). By comparison, the extra dimension acquired from the use of 2D NMR logging methods enables the construction of a 2D map of the proton distribution function of $\left(T_{2}, D\right)$ or $\left(T_{2}, T_{1}\right)$, which is capable of handling complex reservoir fluid typing and evaluation.

Table 1 NMR properties of fluids for a typical sandstone reservoir ( $G=17$ Gauss $/ \mathrm{cm}, T E=1.2 \mathrm{~ms}$ ) (Dunn et al, 2002)

\begin{tabular}{ccccc}
\hline Fluid & $\begin{array}{c}T_{1} \\
\mathrm{~ms}\end{array}$ & $\begin{array}{c}T_{2} \\
\mathrm{~ms}\end{array}$ & $\begin{array}{c}D \\
\times 10^{-5} \mathrm{~cm}^{2} / \mathrm{s}\end{array}$ & $\begin{array}{c}\frac{1}{12} \gamma^{2} G^{2} D(T E)^{2} \\
\mathrm{~s}^{-1}\end{array}$ \\
\hline Brine & $1-500$ & $0.67-200$ & 7.7 & 1.9 \\
Oil & 1000 & 670 & 2 & 0.5 \\
Gas & 4000 & 40 & 100 & 24.8 \\
\hline
\end{tabular}

\section{One-dimensional NMR logging typing flu- id methods}

\subsection{Dual $T W$ method}

Oil and gas are usually nonwetting phases. Their $T_{1} \mathrm{~s}$ are much longer than the $T_{1}$ of water in the rock. Dual $T W$ uses different waiting time for full polarization of oil, gas and water. Two sets of echo trains are acquired in a long or short waiting time independently. Short waiting time $\left(T W_{\mathrm{s}}\right)$ is shorter than $T_{1, \mathrm{gas}}$ for gas, or $T_{1, \mathrm{oil}}$ for oil, but longer than three times the longest $T_{1 \mathrm{w}}$ of the water contained in rock and ensures full recovery for water, and long waiting time $\left(T W_{\mathrm{L}}\right)$ is long enough for recovering all gas and oil signals in the following (Dunn et al, 2002):

$$
3 T_{1 \mathrm{w}, \text { max }}<T W_{\mathrm{S}}<T_{1, \text { gas }}\left(\text { and } / \text { or } T_{1, \text { oil }}\right)<T W_{\mathrm{L}}
$$

NMR $T_{2}$ relaxation signals measured with long and short periods of waiting time are shown independently in the following forms:

$$
\begin{aligned}
& \phi_{T W_{\mathrm{L}}}=\phi_{\mathrm{w}}+\phi_{\text {gas }}\left(1-\mathrm{e}^{-T W_{\mathrm{L}} / T_{1, \text { gas }}}\right)+\phi_{\text {oil }}\left(1-\mathrm{e}^{-T W_{\mathrm{L}} / T_{1, \mathrm{oil}}}\right) \\
& \phi_{T W_{\mathrm{S}}}=\phi_{\mathrm{w}}+\phi_{\text {gas }}\left(1-\mathrm{e}^{-T W_{\mathrm{S}} / T_{1, \text { gas }}}\right)+\phi_{\text {oil }}\left(1-\mathrm{e}^{-T W_{\mathrm{S}} / T_{1, \text { oil }}}\right)
\end{aligned}
$$

Eq. (3) is subtracted with Eq. (2)

$$
\begin{aligned}
\phi_{T W_{\mathrm{L}}}-\phi_{T W_{\mathrm{S}}}= & \phi_{\text {gas }}\left(\mathrm{e}^{-T W_{\mathrm{S}} / T_{1, \text { gas }}}-\mathrm{e}^{-T W_{\mathrm{L}} / T_{1, \text { gas }}}\right) \\
& +\phi_{\text {oil }}\left(\mathrm{e}^{-T W_{\mathrm{S}} / T_{1, \text { il }}}-\mathrm{e}^{-T W_{\mathrm{L}} / T_{1, \text { oil }}}\right)
\end{aligned}
$$

where $\phi_{T W_{\mathrm{L}}}$ and $\phi_{T W_{\mathrm{S}}}$ represent the $T_{2}$ signals for long and short periods of waiting time, respectively, and $\phi_{\mathrm{w}}$ for water signal, $\phi_{\text {gas }}$ for gas signal, and $\phi_{\text {oil }}$ for oil signal. The factors, $\mathrm{e}^{-T W_{\mathrm{L}} / T_{1, \text { gas }}}$ and $\mathrm{e}^{-T W_{\mathrm{L}} / T_{1, \text { oil }}}$, are very small and can be omitted. Eq.(4) becomes (Dunn et al, 2002):

$$
\phi_{T W_{\mathrm{L}}}-\phi_{T W_{\mathrm{S}}}=\phi_{\mathrm{gas}} \mathrm{e}^{-T W_{\mathrm{S}} / T_{1, \text { gas }}}+\phi_{\text {oil }} \mathrm{e}^{-T W_{\mathrm{S}} / T_{1, \text { oil }}}
$$

From Eq. (5), oil and gas saturations can be obtained. The success of the application of this method depends on NMR relaxation contrast between water and light hydrocarbons, physical properties of reservoir rock, and NMR logging acquisition parameter. Dual $T W$ has favourable results of typing gas and light oil (viscosity within 1-5 mPa.S) (Coates et al, 1999). When water zones with macropores are present in a reservoir, the presence of water signals in the differential spectrum could result in erroneous interpretation. Fig. 1 is an example of NMR Dual $T W$ logging from an oilfield. $T W_{\mathrm{L}}$ is $13 \mathrm{~s}$, and $T W_{\mathrm{S}}$ is $1 \mathrm{~s}$. The differential spectrum signals appeared in every zone in the figure (the eighth channel), but production test results (the ninth channel) indicate that they were water zones. The macropore throats are quite open, leading to a large $T_{1}$ for water. The short waiting time of $1 \mathrm{~s}$ was not sufficient for the water to fully recover, which resulted in the appearance of water signals in Eq. (5).

\subsection{Dual TE method}

The dual TE method is based on different diffusion coefficients among oil, gas and water in a constant field gradient. The shift of $T_{2}$ amplitude distributions to shorter relaxation time is different for different fluids when long and short echo spacings are applied. For water wet rock in a constant field gradient, the $T_{2}$ relaxation rate of water $1 / T_{2, \mathrm{w}}$ is given as follows:

$$
\frac{1}{T_{2, \mathrm{w}}}=\frac{1}{T_{2 \mathrm{~B}, \mathrm{w}}}+\frac{1}{T_{2 \mathrm{~S}, \mathrm{w}}}+\frac{1}{12} \gamma^{2} G^{2} D_{\mathrm{w}}(T E)^{2}
$$

where $T_{2 \mathrm{~B}, \mathrm{w}}$ is the $T_{2}$ of bulk water, $T_{2 \mathrm{~S}, \mathrm{w}}$ the $T_{2}$ of surface relaxation, $G$ the constant field gradient, $D_{\mathrm{w}}$ the self diffusion coefficient of water, $T E$ the spacing (or time) between echoes, $\gamma$ the proton gyromagnetic ratio. By increasing $T E$, the $T_{2}$ amplitude distributions shift to a shorter relaxation time. Since the increase of the relaxation rate (the third term in Eq. (6)) is a constant, the shift of $T_{2}$ because of this diffusion term will become larger at long relaxation time but quite small at short relaxation time (Dunn et al, 2002).

Because oil is usually a nonwetting phase, its $1 / T_{2,0}$ depends on the bulk $T_{2 \mathrm{~B}, 0}$, diffusion coefficients $D_{0}$, the echo spacing $T E$, and the gradient strength $G$, i.e.:

$$
\frac{1}{T_{2, \mathrm{o}}}=\frac{1}{T_{2 \mathrm{~B}, \mathrm{o}}}+\frac{1}{12} \gamma^{2} G^{2} D_{\mathrm{o}}(T E)^{2}
$$




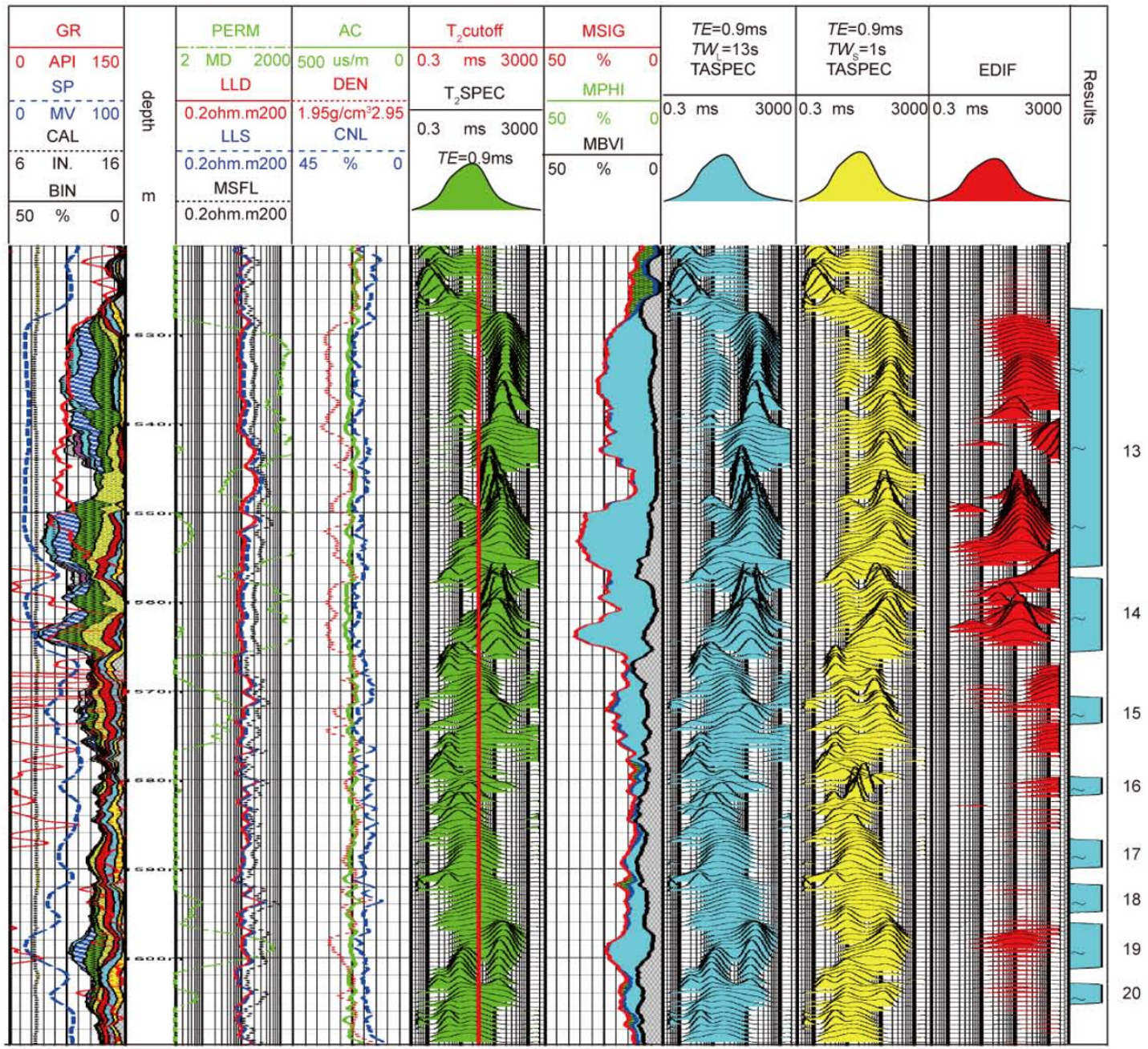

Fig. 1 An example for differential spectrum signals in water zones with Dual $T W$ measurements

Notes: In this figure, the first channel includes gamma ray log, GR (red solid line), spontaneous potential log, SP (blue long dashed line), caliper log (black dotted line) and $T_{2}$ binary digit from long $T W$; The second channel includes the permeability curve, PERM (green dot dashed line), deep lateral resistivity log, LLD (red solid line), shallow lateral resistivity log, LLS (blue long dashed line) and microresistivity log, MSFL (black dotted line). The third channel includes sonic log, AC (green solid line), density $\log$, DEN (red dotted line) and neutron log, CNL (blue long dashed line). The fourth channel includes $T_{2}$ cutoff (red solid line) and standard $T_{2}$ spectrum. The fifth includes total porosity, MSIG (red solid line), effective porosity, MPHI (green solid line) and capillary bound water porosity, MBVI (black solid line). The sixth channel is the long $T W T_{2}$ spectrum. The seventh channel represent

the short $T W T_{2}$ spectrum. The eighth channel is the differential spectrum. The ninth channel is the production test result.

The diffusion coefficient of water, $D_{\mathrm{w}}$ is usually an order of magnitude higher than that of oil. Thus the diffusion term in the relaxation rate equation Eqs. (6) and (7) for water is much larger than that for oil. If the signals for water and oil overlap initially, a larger $T E$ can often produce sufficient relative shift in $T_{2}$ and hence can help identify both oil and water signals. The diffusion coefficient of gas is often two orders of magnitude larger than that of water. The $T_{2}$ for gas is in the neighborhood of $40 \mathrm{~ms}$ as shown in Table 1. Further increase in $T E$ would shift the gas signal to the region of irreducible water. Besides, it is very hard to detect the effect of varying $T E$ because the gas signal is very weak (Dunn et al, 2002). Hence, Dual TE is often used for typing oil and water, while dual $T W$ can be used for oil, gas and water.

\section{Two-dimensional NMR logging typing fluid methods}

\section{1 $\left(T_{2}, D\right)$ method}

The new generation of NMR logging tools can acquire spin echo trains of multiple TEs. The echo amplitude $b(t, T E)$ can be shown as follows (Depavia et al, 2003; Chen et al, 2003; Xie, 2008):

$$
b(t, T E)=\iint f\left(T_{2}, D\right) \mathrm{e}^{-t / T_{2}} \mathrm{e}^{-\frac{1}{12} \gamma^{2} G^{2}(T E)^{2} D t} \mathrm{~d} D \mathrm{~d} T_{2}+\varepsilon
$$

where $b(t, T E)$ is the echo amplitude, $f\left(T_{2}, D\right)$ the two dimensional function, $\gamma$ the gyromagnetic ratio. $G$ the con- 
stant magnetic field gradient, $D$ the diffusion coefficient, $T E$ the time between echoes and $\varepsilon$ is the noise.

In the discrete form, Eq.(8) can be written as:

$$
b_{i k}=\sum_{j=1}^{m} \sum_{l=1}^{p} f_{j l} \mathrm{e}^{-t_{i} / T_{2 j}} \mathrm{e}^{-\frac{1}{12} \gamma^{2} G^{2}\left(T E_{k}\right)^{2} D_{l} t_{i}}+\varepsilon_{i k}
$$

where $b_{i k}$ is the amplitude of $i$-th echo using $T E_{k}, f_{j l}$ the number of hydrogen nuclei with diffusion coefficient $D_{l}$ and at relaxation time $T_{2 j}, \varepsilon_{i k}$ the noise, $m$ the number of relaxation times $T_{2 \mathrm{j}}$, and $p$ the number of diffusion coefficients $D_{\mathrm{l}}$, all equally spaced on their respective logarithmic scales. By inverting Eq.(9), we can obtain the $2 \mathrm{D}$ distribution function $f_{j l}$ (Xie et al, 2009b).

\section{$4.2\left(T_{2}, T_{1}\right)$ method}

The echo amplitude $b(t, T W)$ acquired with multiple periods of waiting time $(T W)$ in a single logging pass can be shown as follows (Xie et al, 2009a; Xie, 2008; Xie et al, 2009b):

$$
b(t, T W)=\iint f\left(T_{2}, T_{1}\right) \mathrm{e}^{-t / T_{2}}\left(1-\mathrm{e}^{-T W / T_{1}}\right) \mathrm{d} T_{2} \mathrm{~d} T_{1}+\varepsilon
$$

where $b(t, T W)$ is the echo amplitude, $f\left(T_{2}, T_{1}\right)$ a $2 \mathrm{D}$ function, $T W$ the waiting time between successive CPMG excitations, and $\varepsilon$ is the noise.

In the discrete form, Eq.(10) can be written as:

$$
b_{i k}=\sum_{j=1}^{m} \sum_{r=1}^{n} f_{j r} \mathrm{e}^{-t_{i} / T_{2 j}}\left(1-\mathrm{e}^{-T W_{k} / T_{1 r}}\right)+\varepsilon_{i k}
$$

where $b_{i k}$ is the amplitude of $i$-th echo using $T W_{k}, f_{j r}$ the number of hydrogen nuclei at transverse relaxation time $T_{2 j}$ and longitudinal relaxation time $T_{1 r}$, and $\varepsilon_{i k}$ the noise. $m$ is the preset number of $T_{2 j}$ relaxation time, $n$ also the preset number of $T_{1}$, relaxation time assumed by the model, both equally spaced on their respective logarithmic scales. By inverting Eq.(11), we can obtain the 2D distribution function $f_{j r}$ (Xie et al, 2009b).

\subsection{Numerical simulation and result analysis}

2D NMR logging can provide more information on multiple periods of waiting time or echo spacing time than $1 \mathrm{D}$ NMR logging in a single logging pass, both of which have been shown to greatly improve the capability of hydrocarbon typing (Xie et al, 2009a; Xie and Xiao, 2009). In this work, two kinds of reservoir are taken into consideration: gasbearing water layer with movable water in big pores and oilbearing water layer with movable water in big pores. These two different situations are simulated respectively in order to study the capability of 2D NMR logging fluid typing methods concerning the complex effect of both macroporous structures and different reservoir fluids.

Suppose the gas-bearing water layer is composed of irreducible water and gas and movable water in big pores, the distribution of each component in the $\left(T_{2}, D\right)$ chart assumes a Gaussian shape. We set the $T_{2}$ relaxation of irreducible water to be $10 \mathrm{~ms}$ and $T_{1}$ to be $30 \mathrm{~ms}$ with a saturation of $40 \%$; the $T_{2}$ of movable water in big pores to be $1000 \mathrm{~ms}$ and $T_{1}$ to be $2000 \mathrm{~ms}$ with a saturation of $30 \%$, the diffusion coefficient $D$ to be $2.5 \times 10^{-5} \mathrm{~cm}^{2} / \mathrm{s}$ for both irreducible water and movable water; and the $T_{2}$ of gas to be $40 \mathrm{~ms}, T_{1}$ to be $3000 \mathrm{~ms}$ and $D$ to be $1 \times 10^{-3} \mathrm{~cm}^{2} / \mathrm{s}$ with a gas saturation of $30 \%$. The field gradient $G$ used in the simulation is $0.35 \mathrm{~T} / \mathrm{m}$. Fifteen sets of echo trains are generated with the time of echo spacings being respectively $0.2,0.3,0.4,0.5,0.6,0.7,0.8,0.9,1.0$, $1.2,1.6,2.0,2.4,4.8$ and $9.6 \mathrm{~ms}$. Fig. 2 illustrates the $\left(T_{2}\right.$,
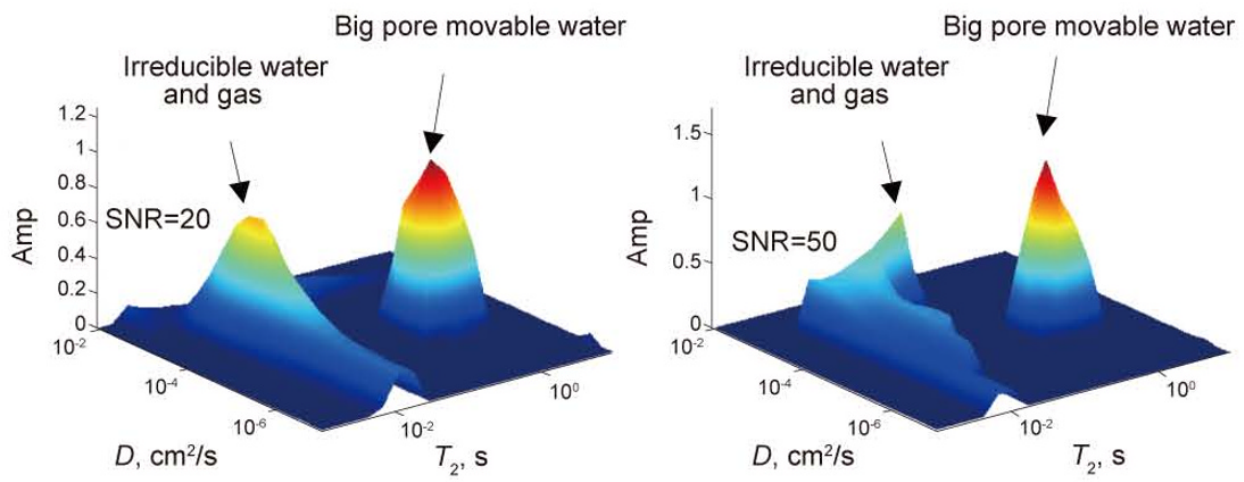

Big pore movable water
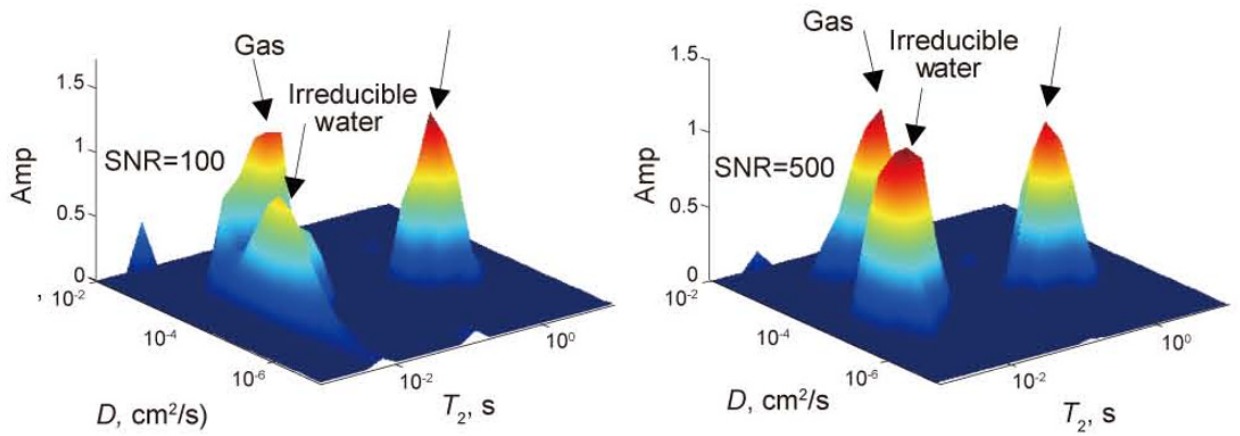

Fig. $2\left(T_{2}, D\right)$ two-dimensional inversion results of a gas-bearing water layer with movable water in big pores at different SNRs 
$D$ ) inversion results for the gas-bearing water layer with movable water in big pores at different SNRs with fixed $G$ $0.35 \mathrm{~T} / \mathrm{m}$. It shows that the $\left(T_{2}, D\right)$ method for fluid typing becomes much easier with the increase of SNR. Even though the SNR is less than 20 , the movable water in big pores can be clearly distinguished. When SNR is less than 50 , the $\left(T_{2}\right.$, $D)$ method cannot separate the irreducible water from gas. With the increase of SNR, the effectiveness of the $\left(T_{2}, D\right)$ method for typing irreducible water and gas can be further improved, which can differentiate the overlapping $T_{2}$ signals of irreducible water and gas encountered in the use of the dual TE method in 1D NMR.

Fifteen sets of echo trains are generated with the length of waiting time being $10,30,50,70,100,300,500,700,1000$, 2000, 4000, 8000, 10000, 12000 and 15000 ms. Fig. 3 shows the $\left(T_{2}, T_{1}\right)$ inversion results for this gas-bearing water layer with movable water in big pores at different SNRs. It shows that the $\left(T_{2}, T_{1}\right)$ method can clearly distinguish gas, irreducible water and movable water in big pores, even though the SNR is less than 20. When Fig. 3 is compared with Fig. 2, the $\left(T_{2}\right.$, $T_{1}$ ) method has more advantages in typing irreducible water and gas than the $\left(T_{2}, D\right)$ method.
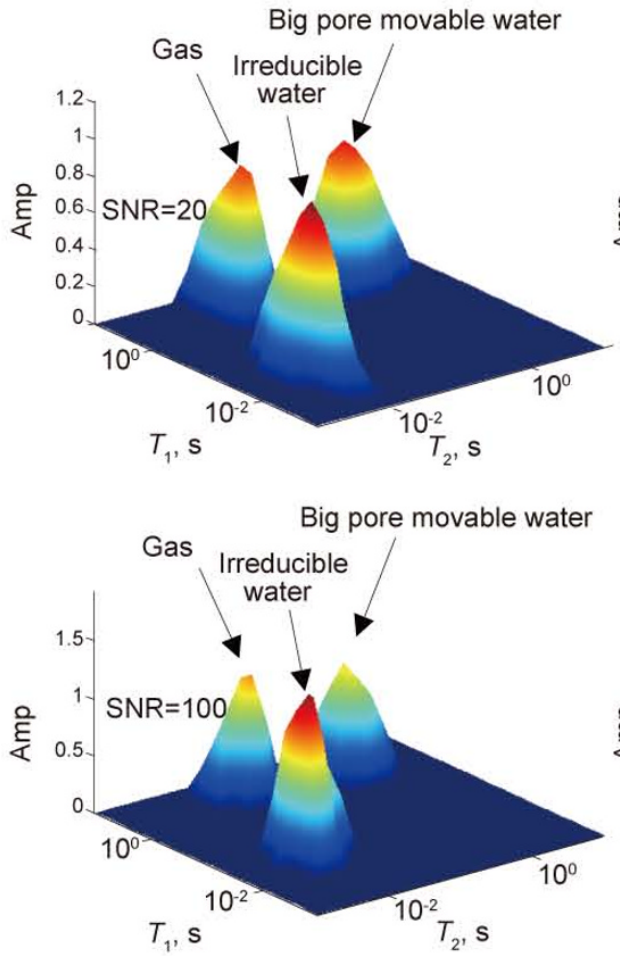

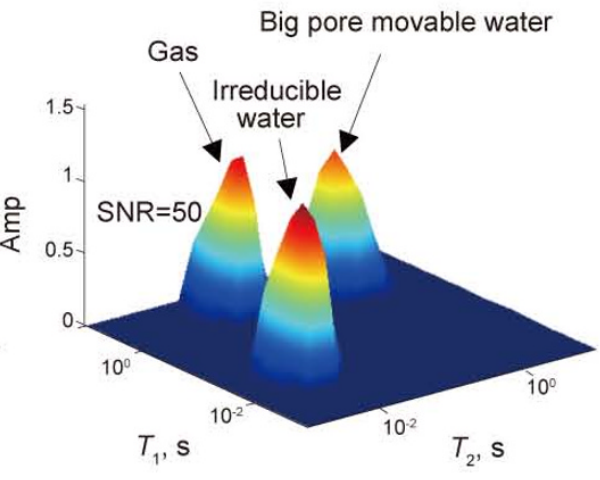

Big pore movable water

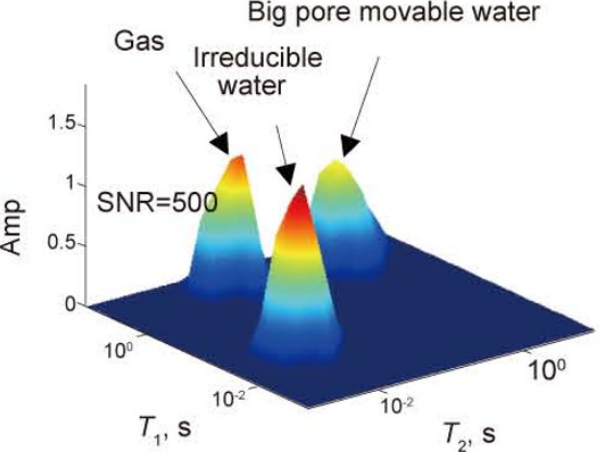

Fig. $3\left(T_{2}, T_{1}\right)$ two-dimensional inversion results of a gas-bearing water layer with movable water in big pores at different SNRs

In a different situation, instead of gas we use medium viscosity oil with $T_{2}$ being $200 \mathrm{~ms}$ and keep the $T_{2}, T_{1}$ and $D$ of irreducible water and movable water unchanged in big pores in the above model. The estimated viscosity of oil is about $14.7 \mathrm{mPa} \cdot \mathrm{s}$ at $35^{\circ} \mathrm{C}$ using the equation established by Lo et al (2000). Suppose the oil bearing water layer is composed of irreducible water, big pore movable water and oil. Assuming that the $T_{1}$ of oil is $400 \mathrm{~ms}, D$ being $2 \times 10^{-6} \mathrm{~cm}^{2} / \mathrm{s}$ with oil saturation of $30 \%$, fifteen sets of echo trains are generated with the time of echo spacings being $0.2,0.3,0.4,0.5,0.6$, $0.7,0.8,0.9,1.0,1.2,1.6,2.0,2.4,4.8$ and $9.6 \mathrm{~ms}$ with the simulated field gradient $G$ being $0.35 \mathrm{~T} / \mathrm{m}$. Fig. 4 illustrates the $\left(T_{2}, D\right)$ inversion results for the layer at different SNRs. It shows that the $\left(T_{2}, D\right)$ method can distinguish irreducible water, movable water in big pores and medium viscosity oil, even though the SNR is less than 20. The effectiveness of the $\left(T_{2}, D\right)$ method for typing fluids becomes better with an increase of SNR. Comparing Fig. 4 with Fig. 2, the $\left(T_{2}, D\right)$ method has more advantages in typing an oil-bearing water layer than a gas-bearing water layer when the SNR of data is low.

Fifteen sets of echo trains are generated with waiting times of 10, 30, 50, 70, 100, 300, 500, 700, 1000, 2000, $4000,8000,10000,12000$ and $15000 \mathrm{~ms}$. Fig. 5 shows the $\left(T_{2}, T_{1}\right)$ inversion results for this oil-bearing water layer with movable water in big pores at different SNRs. The results indicate that the $\left(T_{2}, T_{1}\right)$ method can distinguish irreducible water, movable water and oil as long as the difference of $T_{2}$ between the movable water and oil is sufficiently large. Thus, the $\left(T_{2}, T_{1}\right)$ method can resolve this particular situation where the dual $T W$ method often mistakes water in big pores for oil. The $\left(T_{2}, T_{1}\right)$ method can distinguish irreducible water and medium viscosity oil with a wider viscosity range than can be resolved by the dual $T W$ method. 

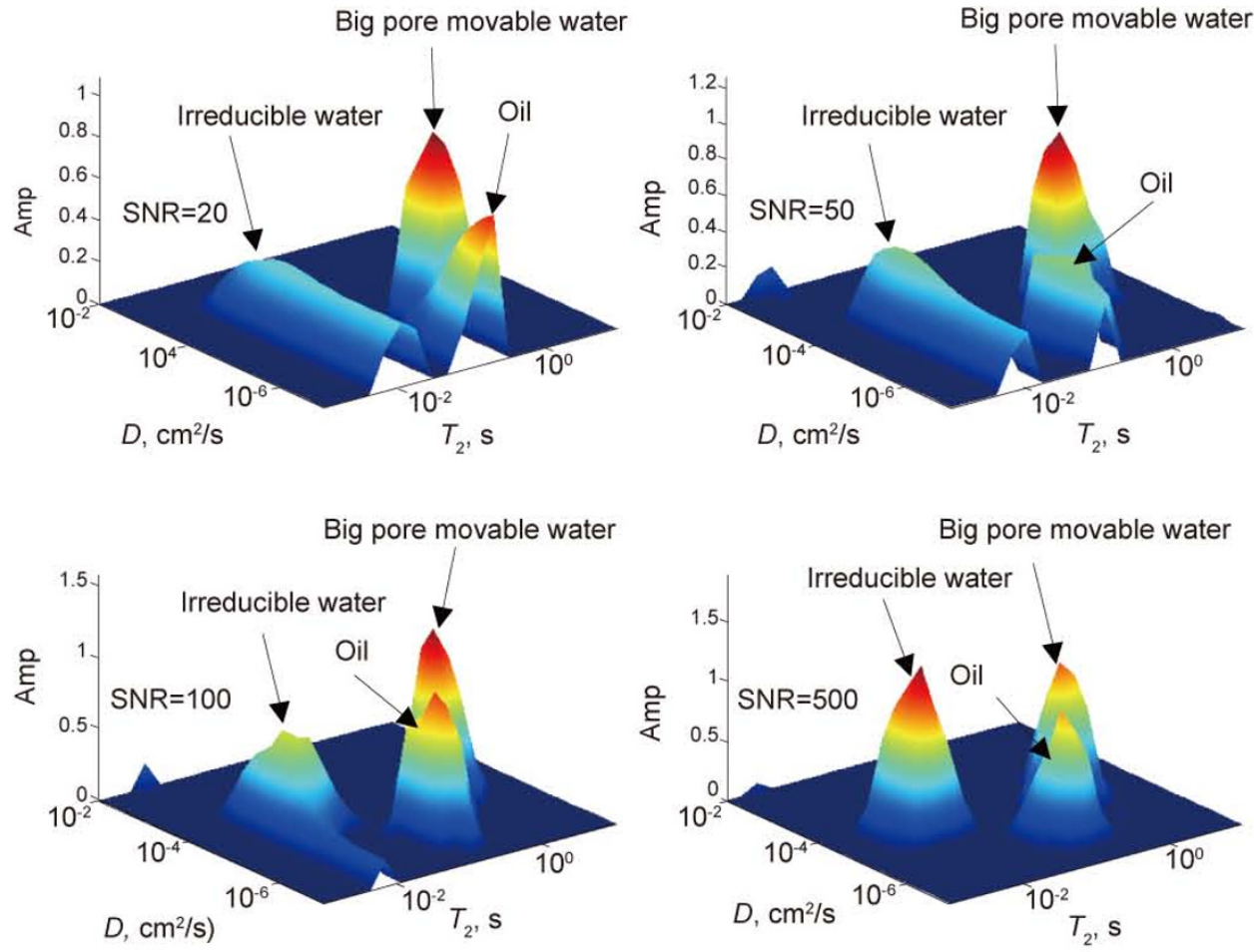

Fig. $4\left(T_{2}, D\right)$ two-dimensional inversion results of an oil-bearing water layer with movable water in big pores at different SNRs
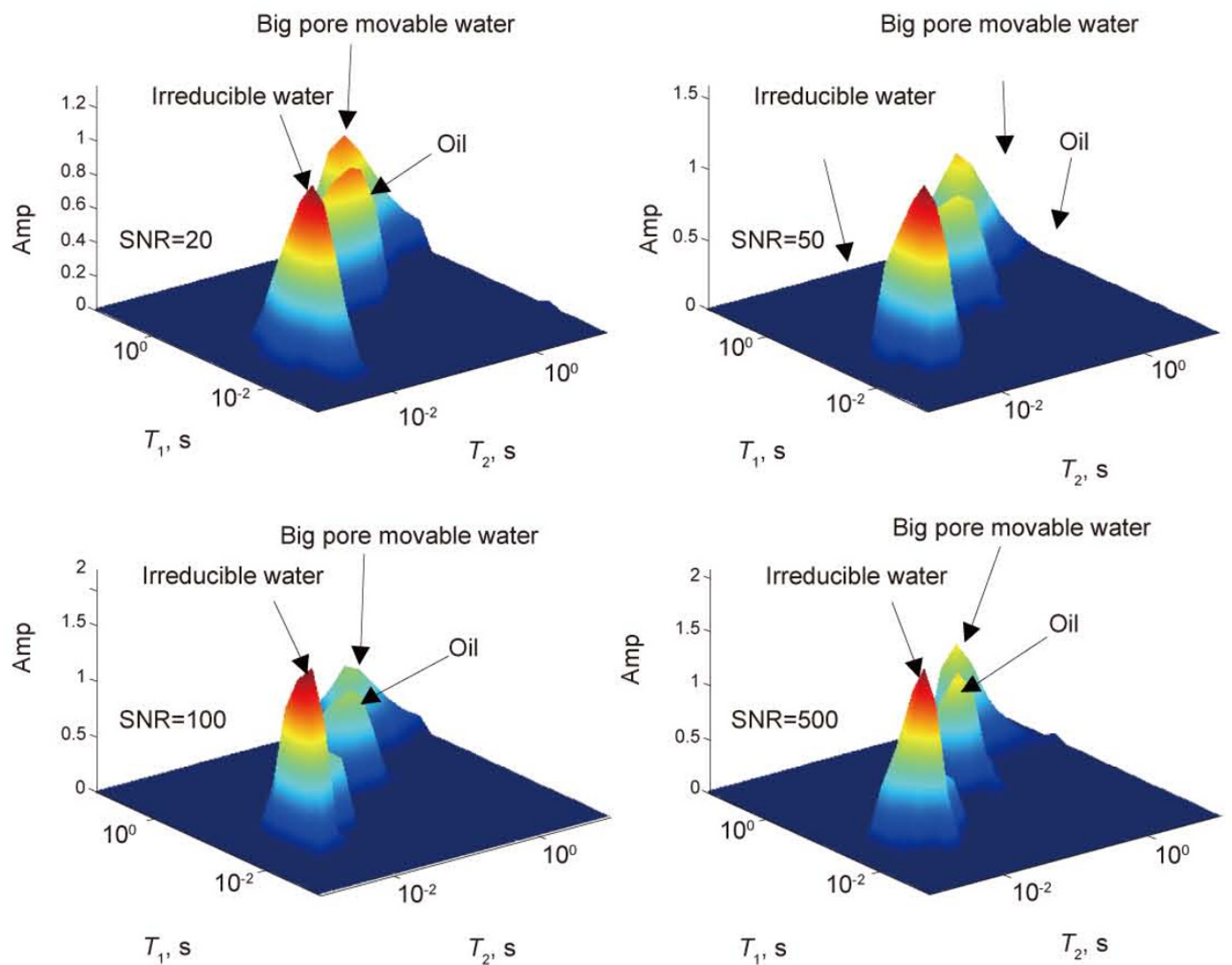

Fig. $5\left(T_{2}, T_{1}\right)$ two-dimensional inversion results of an oil-bearing water layer with movable water in big pores at different SNRs 


\section{Conclusions}

1) The dual $T W$ method is effective in typing gas and light oil (viscosity from $1 \mathrm{mPa} \cdot \mathrm{s}$ to $5 \mathrm{mPa} \cdot \mathrm{S}$ ). When water zones with big pores are present in a reservoir, the water signal will appear in differential spectra in the water zone due to the insufficiency of the short waiting time, resulting in an incorrect interpretation of a water layer as an oil layer.

2) The dual $T E$ method is not suitable for typing gas and irreducible water because of the $T_{2}$ signals of irreducible water significantly overlapping that of the gas in the 1D $T_{2}$ distribution.

3) The $\left(T_{2}, T_{1}\right)$ method is effective in typing gas layers. In an oil-bearing water layer with movable water in big pores, the $\left(T_{2}, T_{1}\right)$ method can distinguish movable water, oil and irreducible water when their $T_{2}$ distributions are separate, which can solve the problem arising from an incorrect interpretation of a water layer in big pores as an oil layer due to the use of the dual $T W$ method. The $\left(T_{2}, T_{1}\right)$ method can distinguish irreducible water from medium viscosity oil and as a result, the viscosity range of oil which can be identified becomes wide in contrast with that of the dual $T W$ method.

4) The $\left(T_{2}, D\right)$ method is more effective than $\left(T_{2}, T_{1}\right)$ in tying an oil and water layer. In a gas layer, when the SNR is higher than a threshold, the $\left(T_{2}, D\right)$ method can resolve the problem of the overlapping $T_{2}$ signals of irreducible water and gas for the dual $T E$ method which is encountered in 1D NMR.

$5)$ Both the $\left(T_{2}, D\right)$ and $\left(T_{2}, T_{1}\right)$ methods use the contrasts in relaxation time and diffusion coefficients between water and hydrocarbon. They significantly improve the capability of fluids typing. This is the future direction of NMR logging for typing complex reservoir fluids.

\section{Acknowledgements}

The authors acknowledge the financial support from PetroChina Company Limited Innovation Foundation (Grant No.07-06D-01-04-01-07) and State Key Laboratory of Petroleum Resource and Prospecting, China University of Petroleum (Beijing) (Grant No. PRPDX2008-02).

\section{References}

Akkurt R, Vinegar H J, Tutunjian P N, et al. NMR logging of natural gas reservoirs. 36th Annual Logging Symposium Transactions: The Society of Professional Well Log Analysts. 1995. Paper N

Chen S, Beard D, Gillen M, et al. MR explorer log acquisition methods: petrophysical objective oriented approaches. 44th Annual Logging Symposium Transactions: The Society of Professional Well Log Analysts. 2003, Paper: ZZ

Coates G R, Xiao L Z and Prammer M G. NMR Logging Principles and Applications. Texas: Gulf Publishing Company. 1999.

DePavia L, Heaton N, Ayers D, et al. A next-generation wireline NMR logging tool. Society of Petroleum Engineers. 2003. Paper: SPE84482

Dunn K J, Bergman D J and Latorraca G A. Nuclear Magnetic Resonance: Petrophysical and Logging Applications. Pergamon: Elsevier Science. 2002.
He Y D, Mao Z Q, Xiao L Z, et al. An improved method of using NMR $T_{2}$ distribution to evaluate pore size distribution. Chinese J. Geophys. 2005. 48(2): 373-378 (in Chinese)

Hurlimann M D, Venkataramanan L, Flaum C, et al. DiffusionEditing: New NMR measurement of saturation and pore geometry. 43th43rd Annual Logging Symposium Transactions: The Society of Professional Well Log Analysts. Oiso, Japan, June 3-6, 2002. Paper FFF

Lo S W, Hirasaki G J, House W V, et al. Mixing rules and correlations of NMR relaxation time with viscosity, diffusivity, and gas/oil ratio of methane/hydrocarbon mixtures. Society of Petroleum Engineers. 2000. Paper: SPE63217

Lu Wendong, Xiao Lizhi, Li Wei, et al. An application of NMR logging to calculating permeability in low porosity and low permeability reservoirs. China Offshore Oil and Gas. 2007. 19(2): 103-106 (in Chinese)

Prammer M G, Mardon D, Coates G R, et al. Lithology-independent gas detection by gradient-NMR logging. SPE Annual Technical Conference and Exhibition Proceedings, v. $\Omega$ (Formation evaluation and reservoir geology). 1995. SPE30562: 325-336

Song Y Q, Venkataramanan L, Hurlimann M D, et al. T1-T2 correlation spectra obtained using a fast two-dimensional Laplace inversion. Journal of Magnetic Resonance. 2002. 15(4): 261-268

Sun B and Dunn K J. Core analysis with two dimensional NMR. International Symposium of the Society of Core Analysts. Monterey. 2002. SCA 38

Sun B, Dunn K J, Bilodeau B J, et al. Two-dimensional NMR logging and field test results. 45th Annual Logging Symposium Transactions: The Society of Professional Well Log Analysts. 2004. Paper: KK

Tan M J, Zhao W J and Fan Y R. Identification of fluid property with NMR dual-TW well logging data. Natural Gas Industry. 2006. 26(4): 38-40 (in Chinese)

Xiao L Z. Some important issues for NMR logging applications in China. Well Logging Technology. 2007. 31(5): 401-407 (in Chinese)

Xie R H and Xiao L Z. The $\left(T_{2}, D\right)$ NMR logging method for fluids characterization. Chinese J. Geophys. 2009. 52(9): 2410-2418(in Chinese)

Xie R H, Xiao L Z and Dunn K J. NMR logging porosity activation and data processing method. Chinese J. Geophys. 2006. 49(5): 15671572 (in Chinese)

Xie R H, Xiao L Z and Liu T D. NMR relaxation properties of crude oils. Journal of Southwest Petroleum University. 2007. 29(5): 21-24 (in Chinese)

Xie R H, Xiao L Z and Lu D W. $\left(T_{2}, T_{1}\right)$ two-dimensional NMR method for fluids typing. Well Logging Technology. 2009a. 33(1): 26-31 (in Chinese)

Xie R H, Xiao L Z, Dunn K J, et al. Two-dimensional NMR logging. Well Logging Technology. 2005. 29(5): 430-434 (in Chinese)

Xie R H, Xiao L Z, Liu J J, et al. A method for multiple echo trains jointing inversion of NMR relaxation measurements. Chinese J. Geophys. 2009b. 52(11): 2913-2919 (in Chinese)

Xie R H, Xiao L Z, Wang Z D, et al. The influence factors of NMR logging porosity in complex fluid reservoirs. Science in China (Series D). 2008. 51(Supp. II): 212-217

Xie R H. Study of NMR Logging Application Fundamentals to Terrestrial Reservoirs and Investigation of 2-D Methods[Ph. D. thesis]. Beijing: China University of Petroleum, 2008 (in Chinese)

Yun H Y and Tan M J. Observation way and analytical approach of dual waiting time by nuclear magnetic resonance logging. Petroleum Geology and Recovery Efficiency. 2006. 13(4): $96-98$ (in Chinese)

(Edited by Yang Lei) 